\title{
On the Use of Locality Sensitive Hashing for Audio Following
}

\author{
Luis F. Guzmán and Antonio Camarena-Ibarrola \\ Universidad Michoacana de San Nicolas de Hidalgo, Morelia, México \\ lfguzman@correo.fie.umich.mx, camarena@umich.mx
}

\begin{abstract}
Audio Following (AF) is the process of mapping a musician's performance, usually in real-time, to a reference performance that is used as a reference. Such base performance is considered a "correct performance" and thus, the live performace must be aligned to it. The objective of AF is to track the musician's position throughout the performance. We present a novel approach to AF that uses a locality sensitive hashing (LSH) based index to perform such task. First, we obtain the Audio Fingerprint (AFP) of the base performance. Then, the obtained AFP is indexed using LSH. Such performance's AFP is used as a reference to align any other performance of the same music. Next, we obtain half-a-second sub-AFP's of the performance being followed and their corresponding positions in the reference AFP are searched for by querying the index. The system was tested on a set of 22 pianists playing music by Chopin with very good results when comparing the obtained alignment with the ideal alignment.
\end{abstract}

Keywords: audio following, proximity index, signal processing.

\section{Introduction}

Digital signal processing along with information-retrieval techniques have acquired much relevance due to the high amount of problems that benefit from their use. One of such problems is AF which basically consists in real-time alignment between a live performance and a base performance used as reference.

\subsection{Previous Work}

Following a musical performance has been an issue of interest for decades due to its interesting applications. Related work dates back to the early 80's when Dannenberg and Vercoe worked independently in real-time automatic accompaniment [1, 2]. In Score Following (SF) the audio signal is aligned in realtime to a symbolic music score, this audio to score alignment is a well studied problem in which mainly two approaches have been used: dynamic programming [1, [2, 3], 4], [5, 6], [7] and stochastic models, usually Hidden Markov Models (HMM's) 8], 9, [10, [11], 12, [13]. Online music tracking systems were also presented in 14 and 15 .

E. Bayro-Corrochano and E. Hancock (Eds.): CIARP 2014, LNCS 8827, pp. 175-182 2014.

(C) Springer International Publishing Switzerland 2014 
The audio features used for extraction vary greatly. Some of the features that have been used in the past are: pitch [2, [3, [8, note onsets [2, 1, signal energy [8], zero crossings [8], spectral information [4, [7], chromagrams [5], [6, multiple tone analysis [11, tempo [12, [13, and others. Kirchhoff et al in [16] tried to determine which audio feature performed best for the task of audio-to-audio alignment. They analysed a great number of audio features including energy, loudness, spectral flatness, Mel Frequency cepstral coefficients, note-onsets, pitch, and chroma-values among others, ultimately reaching the conclusion that "...the choise of suitable features has to depend on the use case at hand."

\subsection{Applications}

Initially, the motivation behind SF was to provide performers with computerassisted automatic accompaniment 1, [2]. Nowadays, SF has found applications in wider domains such as audio indexing and music teaching. Since SF's objective is to track the performer's position in the score and AF's objective is to track the performer's position in the reference performance, AF and SF share most of their possible applications. If the performer's position is known, it can then be used to trigger events at predetermined instants within the performance, these events may be used for different purposes such as enhancing the viewer's experience at a concert (volume changes, lighting effects, fireworks, etc.); providing feedback in a learning environment; or assisting a music player, for instance, by showing the piece of the score that is relevant for him at an specific time.

\subsection{Proposed Schema}

We use two different performances of the same music, one of them as a Reference Performance (RP) to which the other is aligned. The second performance is called Online Performance (OP) since it is not known a-priori but acquired gradually. First, the AFP of the RP is determined. Next, all posible pieces of the AFP corresponding to $500 \mathrm{~ms}$ (sub-AFPs) are inserted in a proximity index. Two consecutive sub-AFPs are highly overlapped, the time interval between a sub-AFP and the next one is barely $11 \mathrm{~ms}$. Once the index is built, the process of following a performance is done by extracting non-overlapping half-a-second sub-AFPs from the OP, then finding the corresponding position of the OP's sub-AFP within the RP's AFP using the proximity index for that matter.

Since comparisons between sub-AFP's can be costly due to the size of the subAFP's and the distance function used to perform such comparisons, a sequential search approach would not meet real-time requirements. We use a proximity index to quickly find the suitable mapping positions for each of OP's sub-AFP. We call this schema Index Audio Following (IAF).

Our approach differs from previous related works not only because we do not use markovian approaches that would make impossible to recover from errors but in the fact that we combine the use of an LSH based proximity index to efficiently perform the alignment with the fact that we toggle bits to better 
handle to better handle proximity, also the fact that we use an entropy-based AFP which is highly robust.

\section{The Audio-Fingerprint}

Entropy is a measure of the amount of information a signal carries. We use an AFP that is based on the idea that it is information what the human brain perceives, so it computes the signal's entropy for each one of the Bark's scale critical bands. The AFP obtains vectors of 24 entropy values. Once these vectors are obtained a binary coding is performed on them. Vectors are compared with the entropy values of the preceding frame, if entropy increased a ' 1 ' is added to the AFP, otherwise, a ' 0 ' is used. The AFP consists of a set of binary vectors that shows how the signal's entropy evolves over time.

\section{The Proximity Index}

We use a proximity index based on LSH [17. because, unlike pivot-based indexes, LSH-based indexes work well in high intrinsic dimension problems, which is our case. The principle behind LSH consist of using a hash function to select a bucket with the expectation that similar elements will be located in the same bucket with high probability. To deal with proximity, several instances of hash tables and hash functions are used in order to increase the probability of finding such similar element in at least one of the hash tables.

The hash function used by LSH is defined in [17] as follows: for an integer $l$ to be specified later, choose $l$ subsets $I_{1}, \ldots, I_{l}$ of $\{1, \ldots, d\}$, where $d$ is the vectors' dimension. Let $p_{\mid I_{j}}$ denote the projection of vector $p$ on the coordinate set $I_{j}$, i.e., we compute $p_{\mid I}$ by selecting the coordinate positions as per $I$ and concatenating the bits in those positions. Denote $g_{j}(p)=p_{\mid I_{j}}$. For preprocessing. We store each $p \in P$ in bucket $g_{j}(p)$, for $j=1, \ldots, l$. Table 1 presents an example of this hash function with $l=2$ and coordinate sets of 3 positions.

Table 1. Example of the use of the Hash function

\begin{tabular}{|c|c|c|}
\hline Binary Vector & 101001100 \\
\hline Bit Coordinate & \multicolumn{2}{|c|}{123456789} \\
\hline Instance & Coordinate Set $I$ & Hash (Bucket) \\
\hline$l_{1}$ & $I_{1}=\{3,6,9\}$ & $h_{1}=110$ \\
\hline$l_{2}$ & $I_{2}=\{1,5,8\}$ & $h_{2}=100$ \\
\hline
\end{tabular}

\section{Design and Implementation}

This section discusses some relevant design issues and describes the general idea of the AF system. 


\subsection{General Design}

The proposed AF schema has the following stages:

- Preprocessing

1. Obtain RP's AFP.

2. Create LSH index with the obtained AFP.

- Following

1. Obtain OP's sub-AFP.

2. Obtain candidate positions by querying the LSH index.

3. Obtain k-Nearest Neighbors (k-NN) list by comparing OP's sub-AFP and candidate positions' sub-AFP's.

4. Select a position from k-NN list as mapping position.

5. Repeat steps $1-4$ until OP is finished.

\subsection{Preprocessing}

Obtain RP's AFP. We use frames of approximately $93 \mathrm{~ms}$ highly overlapped so every $\tilde{1} 2 \mathrm{~ms}$ a new feature vector is obtained.

Indexing RP's AFP. The LSH index is built using two parameters: The number of instances (i.e. number of hash tables) to be used $l$; and the size $n$ of the coordinate sets. Using many instances raises the quality of the index results but also increases memory requirements. Using bigger coordinate sets will create too many buckets $\left(2^{n}\right)$, which means buckets will be less crowded and so less candidates will be obtained. Otherwise, smaller coordinate sets will create more-crowded buckets and more candidates will be obtained. These 2 values, $l$ and $n$, must be calibrated so that a sufficient amount of candidates is checked, maintaining an unhindered following performance, while still performing much less sub-AFP comparisons than the sequential search.

\subsection{Following}

The process of AF consists of the following steps which are repeated until the OP finishes.

Obtain OP's Sub-AFP. As was mentioned in Sect. 1.3, once the RP has been preprocessed, we start the AF process by obtaining non-overlapping halfa-second sub-AFP's from the OP. Since a new vector is generated every $11.6 \mathrm{~ms}$, these half-a-second sub-AFP's contain 43 binary vectors each. We considered the half-a-second resolution to be enough for most applications while still allowing us to perform the AF process in real time. It is important to mention that, while the extracted OP's sub-AFP's are non-overlapping with each other, their obtained points of alignment can be closer within RP's AFP (they could even be the same point). As such, the alignment between RP and OP is performed at a much higher resolution, determined by the overlap between the frames of the extracted RP's AFP. 
Querying the LSH Index. Once we have a sub-AFP with 43 vectors, each of these vectors is taken individualy and used to query the LSH index. As another way to deal with proximity we use 1-bit variations of each query vector and use them to query the index as well. These queries will return all posible positions within RP's AFP where there's a possible match for the OP's sub-AFP.

Obtaining the k-NN List. We reduce the number of candidates to the $k$ most similar sub-AFP's. This is performed by comparing the OP's sub-AFP with all possible candidate sub-AFPs within the RP. To compare sub-AFPs we tried three distance functions: the Hamming distance; the Levenshtein distance; and the Longest Common Subsequence distance.

Selecting a New Position. The last step in the AF process consists of selecting a mapping position from the k-NN list. We assume that we start from the beginning of the OP, so the starting position is $s_{p}=0$. From there, we assume that the music players will never go back in their performance, they can only advance or sustain the position. Therefore, we choose a next mapping position $\left(n_{p}\right)$ that is located forward ahead or repeat the previous mapping position $\left(p_{p}\right)$. So initially, $s_{p}=0=p_{p}$ and from there $n_{p} \geq p_{p}$. We proposed 4 different modes of selecting $n_{p}$. Mode 1 selects the candidate that is closest in time to $p_{p}$. Mode 2 selects the candidate that reports the least distance to the query sub-AFP. Mode 3 is a combination of Modes 1 and 2, it selects the most appropriate candidate from those selected by Mode 1 and 2. Mode 4 uses linear extrapolation to predict where the next mapping position should be and then selects the candidate that is closest to the prediction. Every mode has its own advantages and disadvantages. Mode 1 favors "small steps" and, usually, cannot deal with large "jumps" in the performace or missing parts. Mode 2 can have problems due to the cyclic nature of music, since some parts of the performance may be very similar to other, this mode may "jump" too far ahead. Mode 4 assumes the alignment between RP and $\mathrm{OP}$ is linear so it usually has problems when there are missing/skipped parts or sustains in the performance.

\section{Experiments and Results}

\subsection{The Data Set}

The data set consists of recordings of two excerpts of solo piano music by $\mathrm{F}$. Chopin, an Étude and a Ballade, played by 22 pianists on a computer-monitored grand piano. The same data set has been used before in [7] and [16]. The length of the performances of the Ballade ranged from 1:52 to 2:31 min and the Étude recordings were between 1:10 and 1:34 min in length.

\subsection{Parameter Validation}

There are some parameters that had to be optimized for the system to work accurately. Mainly, choosing which distance function to use; which mode to use 
for selecting a new mapping position; the size $k$ of the $\mathrm{k}$-NN list; the number $l$ of hash tables (instances) to be used when creating the LSH index; and the size $n$ of the coordinate sets $\left(I_{l}\right)$.

Parameters $l$ and $n$ determine the creation of the LSH index which, in turn, determines the quantity of candidates checked for each sub-AFP query. Parameters distance and $k$ determine the resulting $\mathrm{k}-\mathrm{NN}$ list from which a mapping position will be selected. And finally, mode determines the way such mapping position will be chosen.

In order to determine suitable values for these parameters we used a validation technique similar to those used in [6] and [16. In [6], MIDI files were artificially modified to evaluate the alignment between the performnaces obtained after the process of AF. Similarly in [16, an artificial data set was created using a dynamic time-stretching algorithm. We proposed the use of AFPs that are artificially modified, in known predetermined points, in order to simulate situations that might occur during a performance. We used 3 types of modifications:

1. Some vectors were replicated to simulate sustains.

2. Some vectors were removed to simulate skipped/missing sections.

3. We introduced white noise by changing random bits.

Using these synthetic AFPs as ground truth data, we determined suitable ranges of values for our parameters. These ranges are shown in Table 2 .

Table 2. Suitable values for the IAF parameters

\begin{tabular}{|c|c|}
\hline Parameter & Suitable Value \\
\hline$k$ & $\{x \mid x \in N, 15 \leq x \leq 45\}$ \\
\hline distance & Levenshtein, LCS \\
\hline mode & 3,4 \\
\hline$l$ & $\{x \mid x \in N, 50 \leq x \leq 100\}$ \\
\hline$n$ & $\{x \mid x \in N, 13 \leq x \leq 15\}$ \\
\hline
\end{tabular}

\subsection{Results}

We now present the alignments obtained after the AF process and the ideal alignments obtained after manual following for comparison. For this manual audio following, we annotated the note onset times of four recordings: Études $\# 1$, \#2, \#7 and \#22. With these measurements we were able to discover and graph the ideal alignment between any pair of recordings. The slope of the graph represents the relative tempo between the two pieces. Figure 1 shows the alignment obtained when using Étude \#1 as RP and aligning Études \#2, \#7 and \#22 to it. As Fig. 1 shows, the obtained alignment crosses almost every actual alignment point between performances. As to the index' performance, on an average case, only about $25 \%$ of our database elements are compared, with such low number of comparisons we managed to meet real time requirements. We conclude that the results are adequate. 

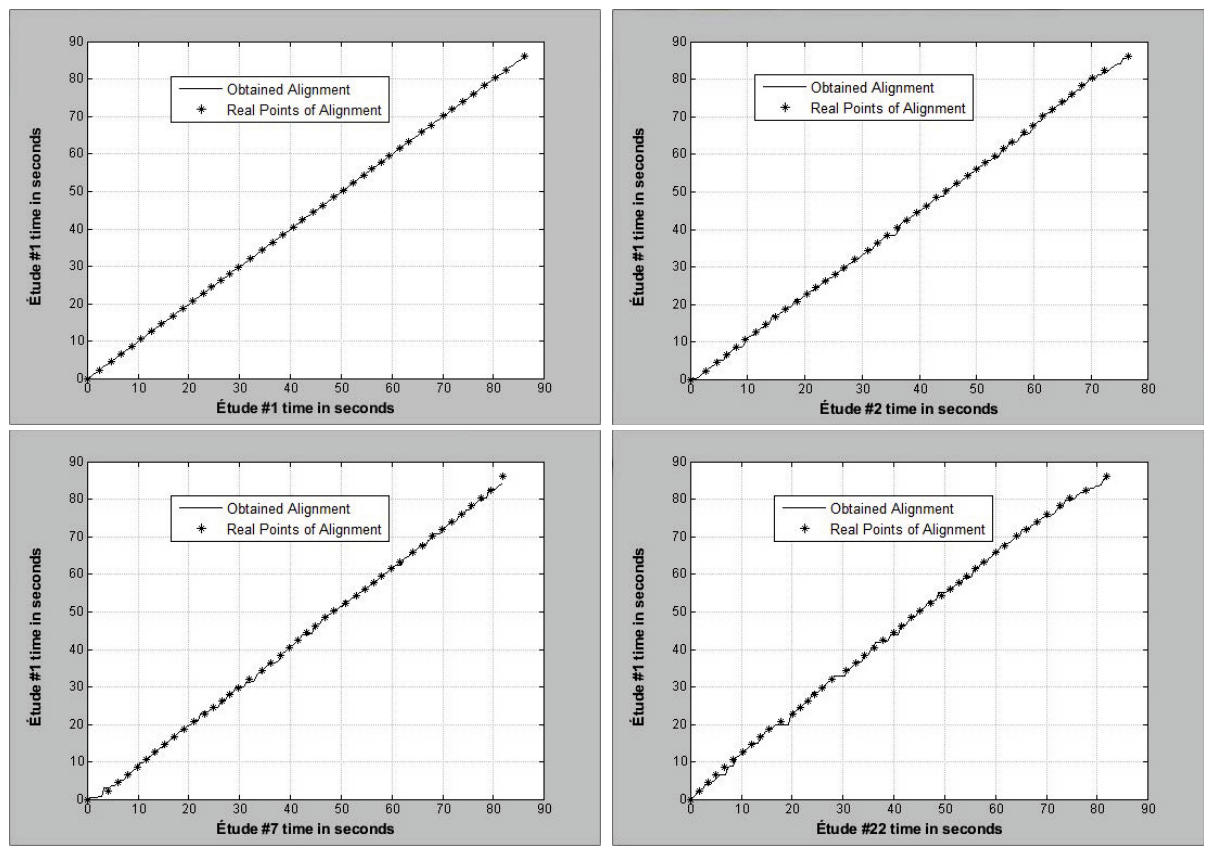

Fig. 1. IAF results using Étude 1 as RP. Versus Études 1 (Top-Left) and 2 (Top-Right). Versus Études 7 (Bottom-Left) and 22 (Bottom-Right).

\section{Conclusions and Future Work}

We proposed a novel approach to perform AF. Using an AFP based on signal entropy and an LSH proximity index, we were able to succesfully map in real time a musical performance to another prepocessed performance of the same music used as a reference. We called this approach Index Audio Following. Experiments were performed using a data set that has been previously used in similar projects [7, 16. Results are encouraging since the obtained alignments are extremely similar to the ideal alignments between performances. However, being the first study to propose this approach, there is still much work to do. As is mentioned in [7, the test recordings were all made under consistent recording conditions (same piano, microfone, room), making the alignment much easier. Further testing using recordings with different characteristics and larger datasets should be done. A method for quantitative evaluation must be develped to replace the qualitative evaluation shown here.

\section{References}

1. Dannenberg, R.B.: An On-line Algorithm for Real-Time Accompaniment. In: Proceedings of the International Computer Music Conference (ICMC), pp. 193-198 (1984) 
2. Vercoe, B.: The Synthetic Performer in the Context of Live Musical Performance. In: Proceedings of the International Computer Music Conference, ICMC (1984)

3. Puckette, M., Lippe, C.: Score Following in Practice. In: Proceedings of International Computer Music Conference, ICMC (1992)

4. Orio, N., Schwarz, D.: Alignment of Monophonic and Polyphonic Music to a Score. In: Proceedings of International Computer Music Conference, ICMC (2001)

5. Hu, N., Dannenberg, R.B., Tzanetakis, G.: Polyphonic Audio Matching and Alignment for Music Retrieval. In: IEEE Workshop on Applications of Signal Processing to Audio and Acoustics (2003)

6. Hu, N., Dannenberg, R.B., Tzanetakis, G.: Polyphonic Audio Matching for Score Following and Intelligent Audio Editors. In: Proceedings of the ICMC, pp. 27-43 (2003)

7. Dixon, S.: Live Tracking of Musical Performances using ON-Line Time Warping. In: 8th International Conference on Digital Audio Effects (2005)

8. Cano, P., Loscos, A., Bonada, J.: Score-Performance Matching using HMMs. In: Proceedings of International Computer Music Conference, ICMC (1999)

9. Raphael, C.: Automatic segmentation of acoustic musical signals using hidden Markov models. IEEE Transactions on Pattern Analysis and Machine Intelligence 21(4), 360-370 (1999)

10. Orio, N., Déchelle, F.: Score Following Using Spectral Analysis and Hidden Markov Models. In: Proceedings of International Computer Music Conference, ICMC (2001)

11. Cont, A.: Realtime Audio to Score Alignment for Polyphonic Music Instruments Using Sparse Non-negative constraints and Hierarchical HMMs. In: IEEE International Conference on Acoustics and Speech Signal Processing, ICASSP (2006)

12. Raphael, C.: Aligning music audio with symbolic scores using a hybrid graphical model. Machine Learning 65, 389-409 (2006)

13. Cont, A.: A coupled duration-focused architecture for realtime music to score alignment. IEEE Transaction on Pattern Analysis and Machine Intelligence 32(6), 974$987(2010)$

14. Camarena-Ibarrola, A., Chávez, E.: Real Time Tracking of Musical Performances. In: Sidorov, G., Hernández Aguirre, A., Reyes García, C.A. (eds.) MICAI 2010, Part II. LNCS, vol. 6438, pp. 138-148. Springer, Heidelberg (2010)

15. Camarena-Ibarrola, A., Chávez, E.: Online music tracking with global alingment. International Journal of Machine Learning and Cybernetics, 147-156 (2011)

16. Kirchhoff, H., Lerch, A.: Evaluation of Features for Audio-to-Audio Alignment. Journal of New Music Research 40(1), 27-41 (2011)

17. Gionis, A., Indyk, P., Motwani, R.: Similarity search in high dimensions via hashing. In: Proceedings of the 25th International Conference on Very Large Databases, vol. 1, pp. 518-529 (1999)

18. Santoyo, F., Chávez, E., Téllez, E.: Compressing Locality-Sensitive Hashing Tables. In: Mexican International Conference on Computer Science (ENC), pp. 41-46 (2013) 